PROCEEDINGS OF THE

AMERICAN MATHEMATICAL SOCIETY

Volume 139, Number 2, February 2011, Pages 675-685

S 0002-9939(2010)10537-3

Article electronically published on August 25, 2010

\title{
NON-NEGATIVE RICCI CURVATURE ON CLOSED MANIFOLDS UNDER RICCI FLOW
}

\author{
DAVI MÁXIMO \\ (Communicated by Richard A. Wentworth)
}

\begin{abstract}
In this short paper we show that non-negative Ricci curvature is not preserved under Ricci flow for closed manifolds of dimensions four and above, strengthening a previous result of Knopf for complete non-compact manifolds of bounded curvature. This brings down to four dimensions a similar result Böhm and Wilking have for dimensions twelve and above. Moreover, the manifolds constructed here are Kähler manifolds and relate to a question raised by Xiuxiong Chen.
\end{abstract}

\section{INTRODUCTION}

Ricci flow is a flow of Riemannian metrics designed to improve a given initial Riemannian metric and has been a powerful tool in understanding geometry and topology of manifolds. A nice and important feature of this flow is that it preserves the "non-negativity" of various curvature conditions.

The flow was introduced on closed manifolds by Richard Hamilton in 20. He proved that non-negative scalar curvature is preserved by the flow in all dimensions. He also showed that the flow preserves non-negative Ricci and non-negative sectional curvatures on three dimensional closed manifolds. Later, in [21, Hamilton proved that Ricci flow also preserves the non-negativity of the Riemann curvature tensor in all dimensions. Also in higher dimensions, independent work of Huisken, Margerin and Nishikawa (see [16, Section 7.1]) demonstrated the invariance of certain sets defined by pinching conditions. Haiwen Chen, in [1], showed that 2-nonnegativity of the Riemann tensor is preserved in all dimensions. In dimension four, Hamilton showed in 22 that positive isotropic curvature is invariant under Ricci flow, and it has recently been proved independently by Brendle and Schoen [5] and by Huy T. Nguyen [25] that this holds in dimensions five and above. In their important paper [5], Brendle and Schoen also proved that each of the properties of $M^{n} \times \mathbb{R}$ and $M^{n} \times \mathbb{R}^{2}$ having non-negative isotropic curvature is preserved by Ricci flow for all $n \geq 4$. Critically, the latter property is weaker than pointwise $\frac{1}{4}$-pinched sectional curvature, but stronger than non-negative sectional curvature. In related work, Brendle found that if $M^{n} \times \mathbb{S}^{2}(1)$ has non-negative isotropic curvature, then this remains so when the metric on $M$ is evolved by the Ricci flow (where $\mathbb{S}^{2}(1)$

Received by the editors November 10, 2009 and, in revised form, April 13, 2010.

2010 Mathematics Subject Classification. Primary 53C44, 53C55, 53C21.

Key words and phrases. Closed 4-manifolds, Kähler manifolds, Ricci curvature, Ricci flow, Kähler-Ricci flow, invariant curvature conditions. 
denotes a two-sphere of constant curvature 1); we refer the reader to 4]. Böhm and Wilking in 3] provided a method for constructing new invariant curvature conditions from known ones. Wilking later announced in [33] an interesting result that provides a unified approach for understanding previously discovered invariant curvature conditions.

Ricci flow was extended to complete non-compact manifolds with bounded curvature by Wan-Xiong Shi in [31] and to Kähler manifolds by Huai-Dong Cao in 9] and by Shigetoshi Bando in [1]. Similar invariant curvature conditions were later found for these flows too. In [30], Shi proved that non-negative Ricci curvature is preserved by the flow on complete manifolds of three dimensions. Bando and Mok, in [27] and [1, proved that non-negative bisectional curvature (which is a stronger assumption than and implies non-negative Ricci curvature; see [19]) on closed Kähler manifolds is also an invariant curvature condition, and this later was extended by Shi in [32] to complete non-compact Kähler manifolds with bounded curvature.

There has also been work showing that some curvature conditions are not preserved in general. In [26, Lei Ni exhibited complete non-compact Riemannian manifolds with bounded non-negative sectional curvature of dimensions four and above where the Ricci flow does not preserve the non-negativity of the sectional curvature. Dan Knopf later showed in 23] examples of complete non-compact Kähler manifolds of bounded curvature and non-negative Ricci curvature whose Kähler-Ricci evolutions immediately acquire Ricci curvature of mixed sign, for each complex dimension above one. Finally, in [2], Böhm and Wilking showed an example of a closed twelve dimensional manifold where Ricci flow evolves an initial homogeneous metric of positive sectional curvature to metrics with mixed Ricci curvature. By taking products with spheres, they settled that positive Ricci curvature is not invariant under the flow for dimensions twelve and above.

Starting on 14] and later with [15], Xiuxiong Chen and Gang Tian answered affirmatively the following important question on Ricci flow: on a compact KählerEinstein manifold, does the Kähler-Ricci flow converge to a Kähler-Einstein metric if the initial metric has positive bisectional curvature? They remarked that what their argument really needed was for the Ricci curvature to be positive along the Kähler-Ricci flow. Since the positivity of Ricci curvature being preserved under the Ricci flow was unknown, they used the fact that the positivity of the bisectional curvature is preserved and that it implies positive Ricci curvature. Since their work, others have tried to remove or weaken this strong assumption of positive bisectional curvature, and we refer the interested reader to [28, [29, [12, [13]. In 1988, Richard Hamilton and Huai-Dong Cao announced a proof that positive orthogonal bisectional curvature is preserved along the flow, and that this suffices to guarantee that non-negative Ricci is preserved. More recently, Xiuxiong Chen published a proof in [12 that non-negative Ricci curvature is preserved on a Kähler solution, assuming its bisectional curvature remains positive as long as it exists. Moreover, under the same assumption, he proved that if the initial Ricci curvature is not positive, then the lower bound of Ricci will increase with time and actually reach zero as time goes to infinity. Motivated by his nice result in [12, Chen asked later in 13 if some form of lower bound of the Ricci curvature would be preserved under Ricci flow at least for closed Kähler manifolds. 
The purpose of this short paper is to show that this is not the case in general. For each complex dimension greater than one, we construct a closed Kähler manifold with non-negative Ricci curvature whose evolution under Ricci flow immediately acquires Ricci curvature of mixed sign. Let $M$ denote $\mathbb{C P}^{2}$ blown up at one point (in the next section, following Calabi in [6, we realize $M$ as the total space of a bundle $\mathbb{C P}^{1} \hookrightarrow M \rightarrow \mathbb{C P}^{1}$; see [17, Section 2.7.2] for more details). We prove the following:

Theorem 1. There exist Kähler metrics on $M$ with non-negative Ricci curvature that immediately acquire mixed Ricci curvature when evolved by Ricci flow.

Remark 2. By taking products of $M$ with spheres, we lower Böhm and Wilking's previous result in 2 to real dimension four and above. Observe that these products will not be Kähler manifolds anymore.

Theorem 1 can actually be generalized to all complex dimensions above one:

Theorem 3. For $n \geq 2$, let $M_{n}$ be $\mathbb{C P}^{n}$ blown up at one point. Then, there exist Kähler metrics on $M_{n}$ with non-negative Ricci curvature that immediately acquire mixed Ricci curvature when evolved by Ricci flow.

Remark 4. Examples found in Theorems 1 and 3 can be thought of as compactifications of the ones found by Knopf in [23].

Remark 5. It is also possible to construct Kähler metrics on $M$ where positive Ricci curvature is preserved by Ricci flow. An example is the soliton metric found independently by Koiso [24] and Cao [7].

Remark 6. A three-manifold $M^{3}$ has non-negative Ricci curvature if and only if $M^{3} \times \mathbb{R}$ has non-negative isotropic curvature. Our result shows that non-negative Ricci curvature does not behave well in higher dimensions, so Brendle and Schoen's observation that non-negative isotropic curvature on $M^{n} \times \mathbb{R}$ is invariant suggests that this property is the "right" generalization to higher dimensions.

The rest of this paper is organized as follows. We first deal with the critical case $n=2$ and begin by proving Theorem 1 in Sections 2 and 3: in Section 2, we review $U(2)$-invariant metrics on $\mathbb{C}^{2} \backslash\{0\}$ and construct an initial metric on $M$ with non-negative Ricci curvature, and in Section 3 the proof of Theorem 1 is finished. Finally, in Section 4 we discuss the general case stated in Theorem 3 .

\section{An initial MetriC OF NON-NEGAtive RiCCI CURVATURE}

In this section we consider rotationally symmetric Kähler metrics on $\mathbb{C}^{2} \backslash\{0\}$ and derive an initial metric of non-negative Ricci curvature on the twisted line bundle $\mathbb{C P}^{1} \hookrightarrow M \rightarrow \mathbb{C P}^{1}$ of Calabi [6, Section 3]. Metrics of this sort were also used by Koiso in 24, Cao in [7, 8] and Feldman, Ilmanen and Knopf in [18] to construct examples of gradient Ricci solitons. For convenience of the reader, we start following Calabi in [6] and realizing $M_{n}$ as the total space of a bundle $\mathbb{C P}^{1} \hookrightarrow M \rightarrow \mathbb{C P}^{n-1}$ (for more details about this construction, see [17, Section 2.7.2]). 
2.1. Calabi's bundle construction. Cover the projective space $\mathbb{C P}^{n-1}$ with its usual $n$ charts $\varphi_{\alpha}: \mathcal{U}_{\alpha} \longrightarrow \mathbb{C}^{n-1}$, where $\mathcal{U}_{\alpha}=\left\{\left[x_{1}: x_{2}: \cdots: x_{n}\right] \in \mathbb{C P}^{n-1}: x_{\alpha} \neq\right.$ $0\}$ and $\varphi_{\alpha}:\left[x_{1}: x_{2}: \cdots: x_{n}\right] \mapsto\left(\frac{x_{1}}{x_{\alpha}}, \ldots, \frac{x_{\alpha-1}}{x_{\alpha}}, \frac{x_{\alpha+1}}{x_{\alpha}}, \ldots, \frac{x_{n}}{x_{\alpha}}\right)$. We then can write $\mathbb{C P}^{n-1}=\left(\bigsqcup_{\alpha=1}^{n} \varphi_{\alpha}\left(\mathcal{U}_{\alpha}\right)\right) / \simeq$, where, for example,

$$
\varphi\left(\mathcal{U}_{1}\right) \ni\left(z_{1}, \ldots, z_{n-1}\right) \simeq\left(\frac{1}{z_{1}}, \frac{z_{2}}{z_{1}}, \ldots, \frac{z_{n-1}}{z_{1}}\right) \in \varphi_{2}\left(\mathcal{U}_{2}\right) .
$$

After formally identifying $\mathbb{C P}^{1}=\mathbb{C} \cup\{\infty\}$, we define for each positive integer $k$ the $k$-twisted bundle

$$
\mathcal{F}_{k}^{n}=\left(\bigsqcup_{\alpha=1}^{n}\left(\mathcal{U}_{\alpha} \times \mathbb{C P}^{1}\right)\right) / \sim
$$

where $\mathcal{U}_{\alpha} \times \mathbb{C P}^{1} \ni\left(\left[x_{1}: \cdots: x_{n}\right] ; \xi\right) \sim\left(\left[y_{1}: \cdots: y_{n}\right], \eta\right) \in U_{\beta} \times \mathbb{C P}^{1}$ if, and only if, $\left[x_{1}: \cdots: x_{n}\right]=\left[y_{1}: \cdots: y_{n}\right]$ and $\eta=\left(\frac{y_{\beta}}{x_{\alpha}}\right)^{k} \xi$. Moreover, let $S_{0}=\left\{\left[x_{1}: \cdots:\right.\right.$ $\left.\left.x_{n}\right] ; 0\right\}$ and $S_{\infty}=\left\{\left[x_{1}: \cdots: x_{n}\right] ; \infty\right\}$, which are two global sections of $\mathcal{F}_{k}^{n}$. Set $\widehat{\mathcal{F}_{k}^{n}}=\mathcal{F}_{k}^{n} \backslash\left(S_{0} \cup S_{\infty}\right)$ and let $\Psi: \mathbb{C}^{n} \backslash\{0\} \longrightarrow \widehat{\mathcal{F}}_{k}^{n}$ be the map

$$
\Psi:\left(x_{1}, x_{2}, \ldots, x_{n}\right) \mapsto\left(\left[x_{1}: \cdots: x_{n}\right] ; x_{\alpha}^{k}\right)
$$

if $x_{\alpha} \neq 0$. Because $\left.\left(\left[x_{1}: \cdots: x_{n}\right] ; x_{\alpha}^{k}\right) \sim\left[x_{1}: \cdots: x_{n}\right] ; x_{\beta}^{k}\right)$ whenever $x_{\alpha} \neq 0$ and $x_{\beta} \neq 0$, the map $\Psi$ is well defined. Furthermore, one can check that $\Psi$ is $k$-to-one and surjective.

In particular, when $k=1, \Psi$ is one-to-one so $\mathcal{F}_{k}^{n}=M_{n}$ can be thought of as $\mathbb{C}^{n} \backslash\{0\}$ with $\mathbb{C P}^{n-1}$, s glued at 0 (the section $S_{0}$ ) and $\infty$ (the section $S_{\infty}$ ). We next look for suitable metrics on $\mathbb{C}^{n} \backslash\{0\}$ that can be extended to $M_{n}$.

2.2. $U(2)$-invariant Kähler metrics on $\mathbb{C}^{2} \backslash\{0\}$. Let $g$ be a $U(2)$-invariant Kähler metric on $\mathbb{C}^{2} \backslash\{0\}$, the latter with complex coordinates $z=\left(z^{1}, z^{2}\right)$. Define $u=\left|z^{1}\right|^{2}, v=\left|z^{2}\right|^{2}$ and $w=u+v$.

Since $g$ is a Kähler metric and the de Rham cohomology group $H^{2}\left(\mathbb{C}^{2} \backslash\{0\}\right)=0$, by the $\partial \bar{\partial}$-lemma one can find a global real smooth function $P: \mathbb{C}^{2} \backslash\{0\} \longrightarrow \mathbb{R}$ such that 1

$$
g_{\alpha \bar{\beta}}=\frac{\partial^{2}}{\partial z^{\alpha} \partial \bar{z}^{\beta}} P .
$$

The further assumption of $g$ being rotationally symmetric allows us to write $P=$ $P(r)$, where $r=\log w$. We then set $\varphi(r)=P_{r}(r)$ (we use subscript for the derivative since later $P$ will be regarded as a function of time as well) and compute from (2.1)

$$
g=\left[e^{-r} \varphi \delta_{\alpha \beta}+e^{-2 r}\left(\varphi_{r}-\varphi\right) \bar{z}^{\alpha} z^{\beta}\right] d z^{\alpha} d \bar{z}^{\beta} .
$$

Moreover 2

$$
\left(\begin{array}{ll}
g_{1 \overline{1}} & g_{1 \overline{2}} \\
g_{2 \overline{1}} & g_{2 \overline{2}}
\end{array}\right)=\frac{1}{w^{2}}\left(\begin{array}{cc}
v \varphi+u \varphi_{r} & \left(\varphi_{r}-\varphi\right) \bar{z}^{1} z^{2} \\
\left(\varphi_{r}-\varphi\right) z^{1} \bar{z}^{2} & u \varphi+v \varphi_{r}
\end{array}\right)
$$

and thus we find that $\operatorname{det}\left(g_{\alpha \bar{\beta}}\right)=e^{-2 r} \varphi \varphi_{r}$. From these two past relations we can rapidly observe that a potential $P$ on $\mathbb{C}^{2} \backslash\{0\}$ gives rise to a Kähler metric as in

\footnotetext{
${ }^{1}$ We are following the notation in [17, Chapter 2].

${ }^{2}$ The matrix $(g)$ is actually a $4 \times 4$ matrix, $(g)=\left(\begin{array}{cc}A & 0 \\ 0 & A\end{array}\right)$, where $A=\left(\begin{array}{ll}g_{1 \overline{1}} & g_{1 \overline{2}} \\ g_{2 \overline{1}} & g_{2 \overline{2}}\end{array}\right)$.
} 
(2.1) if, and only if,

$$
\varphi>0 \text { and } \varphi_{r}>0 .
$$

For computing the Ricci curvature, recall that on Kähler manifolds the complex Ricci tensor $R_{\alpha \bar{\beta}} d z^{\alpha} d z^{\bar{\beta}}$ is given by

$$
R_{\alpha \bar{\beta}}=-\frac{\partial^{2}}{\partial z^{\alpha} \partial \bar{z}^{\beta}} \log \operatorname{det} g .
$$

To put this expression in a more useful form we follow 23. and define

$$
\begin{aligned}
G & =-\log \operatorname{det} g=2 r-\log \varphi-\log \varphi_{r}, \\
\psi & =G_{r}=2-\frac{\varphi_{r}}{\varphi}-\frac{\varphi_{r r}}{\varphi_{r}} .
\end{aligned}
$$

One next calculates

$$
R_{\alpha \bar{\beta}}=e^{-r} \psi \delta_{\alpha \beta}+e^{-2 r}\left(\psi_{r}-\psi\right) \bar{z}^{\alpha} z^{\beta},
$$

and thus Ricci can be represented a: 3

$$
\left(\begin{array}{ll}
R_{1 \overline{1}} & R_{1 \overline{2}} \\
R_{2 \overline{1}} & R_{2 \overline{2}}
\end{array}\right)=\frac{1}{w^{2}}\left(\begin{array}{cc}
v \psi+u \psi_{r} & \left(\psi_{r}-\psi\right) \bar{z}^{1} z^{2} \\
\left(\psi_{r}-\psi\right) z^{1} \bar{z}^{2} & u \psi+v \psi_{r}
\end{array}\right) .
$$

Also as in [23] we will say that a $(1,0)$ tensor $W$ is an eigenvector of the complex Ricci tensor corresponding to the eigenvalue $\lambda$ if $R_{\alpha \bar{\beta}} W^{\alpha}=\lambda g_{\alpha \bar{\beta}} W^{\alpha}$. Hence understood, the eigenvalues of Rc are

$$
\begin{aligned}
& \lambda_{1}=\frac{\psi}{\varphi} \quad \text { with eigenvector } \quad U=\bar{z}^{2} \frac{\partial}{\partial z^{1}}+\bar{z}^{1} \frac{\partial}{\partial z^{1}}, \\
& \lambda_{2}=\frac{\psi_{r}}{\varphi_{r}} \quad \text { with eigenvector } \quad V=z^{1} \frac{\partial}{\partial z^{2}}+z^{2} \frac{\partial}{\partial z^{2}} .
\end{aligned}
$$

2.3. A metric of non-negative Ricci curvature. To find a metric of nonnegative Ricci curvature on a Calabi line bundle $\mathbb{C P}^{1} \hookrightarrow M \rightarrow \mathbb{C P}^{1}$, we want a $U(2)$-invariant Kähler potential $P$ on $\mathbb{C}^{2} \backslash\{0\}$ with the following properties:

(1) $\varphi>0$ everywhere;

(2) $\varphi_{r}>0$ everywhere;

(3) $\psi>0$ everywhere;

(4) $\psi_{r} \geq 0$ everywhere;

(5) $g$ extends smoothly to a complete metric by adding a $\mathbb{C P}^{1}$ at $z=0$; and

(6) $g$ extends smoothly to a complete metric by adding a $\mathbb{C P}^{1}$ at $z=\infty$.

To find such $P$, let us rewrite equation (2.5) as

$$
\left[\log \left(\varphi \varphi_{r}\right)\right]_{r}=a
$$

where $a=2-\psi$. We next observe that we can formally solve the ODE above and find the formal solution $(I(f)$ stands for an antiderivate of $f)$

$$
\varphi(r)=\sqrt{I\left(2 e^{I(a)}\right)} .
$$

In particular, if $a$ is a constant function, we find that $\varphi(r)=\sqrt{\frac{2}{a} e^{a r}+c}$ is a solution for (2.7). This $(a \equiv 1)$ in fact was the $\varphi$ used in 23 and, as shown in this particular

$$
{ }^{3} \text { The matrix }(R c) \text { is actually a } 4 \times 4 \text { matrix, }(\mathrm{Rc})=\left(\begin{array}{cc}
R & 0 \\
0 & R
\end{array}\right) \text {, where } R=\left(\begin{array}{ll}
R_{1 \overline{1}} & R_{1 \overline{2}} \\
R_{2 \overline{1}} & R_{2 \overline{2}}
\end{array}\right) \text {. }
$$




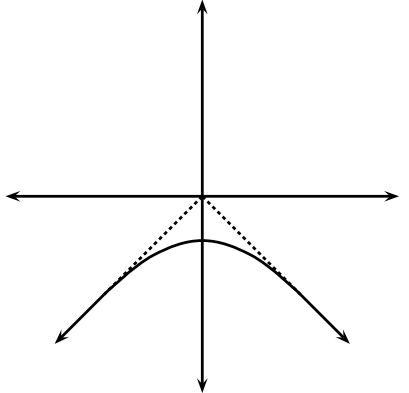

Function $f(r)$

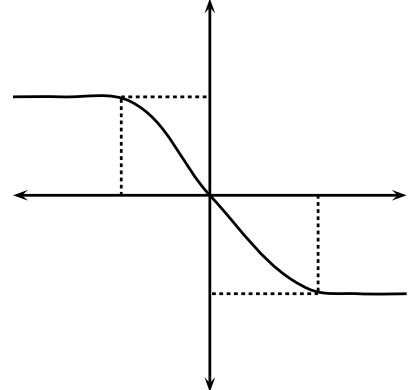

Function $a(r)=f^{\prime}(r)$

Figure 1

reference, it leads to potential $P$ satisfying conditions (1) through (5) above, but not (6).

We consider instead a function $a$ that is constant equal to 1 in a neighborhood of $r=-\infty$, constant equal to -1 in a neighborhood of $r=+\infty$, and smooth on the whole real line.

To be precise, consider the function $y(r)=-|r|$. Because $y^{\prime \prime}(r)=\delta_{0}(r)$, it is possible to smooth $y$ in a small neighborhood of $r=0$ and obtain a smooth function $f(r)$ such that $f^{\prime \prime}(r) \leq 0$. Then, set $a(r)=f^{\prime}(r)$; the graph of the function $a$ is shown on the right in Figure 1.

Since

$$
\int_{\mathbb{R}} e^{f(x)} \mathrm{d} x<\int_{\mathbb{R}} e^{-|x|} \mathrm{d} x<+\infty,
$$

the function $F(r)=\int_{-\infty}^{r} e^{f(x)} \mathrm{d} x$ is well defined and smooth. Thus, we find a solution to equation (2.7) for $a$ chosen as above:

$$
\varphi(r)=\sqrt{2 F(r)+c},
$$

where $c$ is any arbitrary positive constant (since $F(r)>0$ everywhere). We claim that $\varphi$ as above gives a potential $P$ as desired.

First, it is clear that $\varphi$ is positive everywhere, and since $\varphi \varphi_{r}=e^{f}>0, \varphi_{r}$ is also positive everywhere. Thus $\varphi$ satisfies properties (1) and (2) above; i.e., $P$ gives rise to a Kähler metric.

Moreover, $\psi=2-a$, so $\psi>0$ everywhere, since the function $a$ takes values on the interval $[-1,1]$ only. Also (since $f$ is concave) $a$ is non-increasing, so $\psi$ is non-decreasing and thus $\psi_{r} \geq 0$. Hence properties (3) and (4) are also satisfied; that is, $g$ has non-negative Ricci curvature.

To show that $\varphi$ satisfies conditions (5) and (6) it is convenient to compute $F(r)$ when $r$ is near $\pm \infty$ and write explicitly

$$
\begin{aligned}
\varphi=\sqrt{2 e^{r}+c}, & \text { for } r \text { near }-\infty ; \\
\varphi=\sqrt{-2 e^{-r}+c+F_{\infty}}, & \text { for } r \text { near }+\infty,
\end{aligned}
$$

where $F_{\infty}=\int_{\mathbb{R}} e^{f(x)} \mathrm{d} x$ is a constant. 
Calabi's lemma [6. Section 3] tells us that $g$ will extend to a smooth Kähler metric on Calabi's twisted line bundle $\mathbb{C P}^{1} \hookrightarrow M \rightarrow \mathbb{C P}^{1}$ if $\varphi$ satisfies the following asymptotic properties:

(i) There exist positive constants $a_{0}$ and $a_{1}$ such that $\varphi$ has the expansion

$$
\varphi(r)=a_{0}+a_{1} w+a_{2} w^{2}+\mathcal{O}\left(|w|^{3}\right)
$$

as $r \rightarrow-\infty$.

(ii) There exist a positive constant $b_{0}$ and a negative constant $b_{1}$ such that $\varphi$ has the expansion

$$
\varphi(r)=b_{0}+b_{1} w^{-1}+b_{2} w^{-2}+\mathcal{O}\left(|w|^{-3}\right)
$$

as $r \rightarrow \infty$.

For $c>0$, by (2.10), $\varphi$ admits the expansion near $r=-\infty$ :

$$
\varphi=\sqrt{c}+\frac{1}{\sqrt{c}} w-\frac{1}{2 c^{3 / 2}} w^{2}+\mathcal{O}\left(|w|^{3}\right) .
$$

Thus, by Calabi's lemma, property (5) is verified. And near $r=\infty$, by (2.11), $\varphi$ admits the expansion

$$
\varphi=\sqrt{c+F_{\infty}}-\frac{1}{\sqrt{c+F_{\infty}}} w^{-1}-\frac{1}{2\left(c+F_{\infty}\right)^{3 / 2}} w^{-2}+\mathcal{O}\left(|w|^{-3}\right),
$$

so Calabi's lemma again guarantees that $g$ satisfy property (6).

\section{EFFECT OF THE KÄHLER-RICCI FLOW}

We finally consider the Kähler-Ricci flow evolution $(M, g(t))$, where the initial Kähler potential $P(r, 0)$ is taken as the Kähler potential $P(r)$ constructed in the previous section.

Because $g$ is rotationally symmetric, let us work at a point $(\zeta, 0) \in \mathbb{C}^{2} \backslash\{0\}$, where $\zeta \neq 0$ is arbitrary. Since

$$
\left.g\right|_{(\zeta, 0)}=\frac{1}{|\zeta|^{2}}\left(\begin{array}{cc}
\varphi_{r} & 0 \\
0 & \varphi
\end{array}\right)
$$

and

$$
\left.\operatorname{Rc}\right|_{(\zeta, 0)}=\frac{1}{|\zeta|^{2}}\left(\begin{array}{cc}
\psi_{r} & 0 \\
0 & \psi
\end{array}\right)
$$

in the standard basis, the Kähler-Ricci flow equation

$$
\frac{\partial}{\partial t} g=-\mathrm{Rc}
$$

will be satisfied if, and only if, $\varphi$ evolves by $\varphi_{t}=-\psi$. This last assertion is reduced to

$$
\varphi_{t}=\frac{\varphi_{r r}}{\varphi_{r}}+\frac{\varphi_{r}}{\varphi}-2 .
$$

Moreover, the function $\psi$ (since $\frac{\partial}{\partial r}$ and $\frac{\partial}{\partial t}$ commute) must evolve by

$$
\psi_{t}=\frac{\psi_{r r}}{\varphi_{r}}-\frac{\varphi_{r r} \psi_{r}}{\varphi_{r}^{2}}+\frac{\psi_{r}}{\varphi}-\frac{\varphi_{r} \psi}{\varphi^{2}} .
$$

But near $r=-\infty$ we have $\psi(\cdot, 0) \equiv 1$, and this reduces equation (3.2) to

$$
\left.\frac{\partial}{\partial t} \psi\right|_{t=0}=-\frac{\varphi_{r}}{\varphi^{2}}
$$


at the initial time. A final computation then shows that

$$
\left.\frac{\partial}{\partial t} \psi_{r}\right|_{t=0}=\frac{e^{r}}{\varphi^{5}}\left(e^{r}-c\right)
$$

which is strictly negative for $r<\log c$. Hence, the complex Ricci tensor must acquire a negative eigenvalue $\lambda_{2}<0$ at all points close enough to $r=-\infty$. This completes the proof of Theorem 1 .

\section{Complex dimension $n \geq 2$}

In this section we expand our result to higher complex dimensions by proving Theorem 3

If $P$ is now a $U(n)$-invariant Kähler potential on $\mathbb{C}^{n} \backslash\{0\}$, we still have formulas (2.1) and (2.2). The determinant of the matrix $\left(g_{\alpha \bar{\beta}}\right)$ is now $\operatorname{det}\left(g_{\alpha \bar{\beta}}\right)=$ $e^{-n r} \varphi^{n-1} \varphi_{r}$, and, in particular, conditions in (2.3) are still necessary and sufficient. Formula (2.4) then becomes 4

$$
G=n r-(n-1) \log \varphi-\log \varphi_{r},
$$

so (2.5) is replaced by

$$
\psi=G_{r}=n-(n-1) \frac{\varphi_{r}}{\varphi}-\frac{\varphi_{r r}}{\varphi_{r}},
$$

but (2.6) remains the same. By rotational symmetry, we need to display the eigenvalues of Ricci curvature only at points of the form $Z=(\zeta, 0,0, \ldots, 0) \in \mathbb{C}^{n} \backslash\{0\}$, with $\zeta \neq 0$ arbitrary. So we calculate

$$
\left.g\right|_{Z}=\frac{1}{|\zeta|^{2}}\left(\begin{array}{cccc}
\varphi_{r} & & & \\
& \varphi & & \\
& & \ddots & \\
& & & \varphi
\end{array}\right)
$$

and

$$
\left.\mathrm{Rc}\right|_{Z}=\frac{1}{|\zeta|^{2}}=\left(\begin{array}{cccc}
\psi_{r} & & & \\
& \psi & & \\
& & \ddots & \\
& & & \psi
\end{array}\right) .
$$

We next take $\varphi$ as in (2.9). Hence $\varphi$ and $\varphi_{r}$ are both greater than zero everywhere. Moreover, we know that $\psi \equiv n-a$, so $\psi>0$ and, since $a$ is non-increasing, $\psi_{r} \geq 0$.

Furthermore, expansions of $\varphi$,

$$
\varphi=\sqrt{c}+\frac{1}{\sqrt{c}} w-\frac{1}{2 c^{3 / 2}} w^{2}+\mathcal{O}\left(|w|^{3}\right),
$$

near $r=-\infty$, and

$$
\varphi=\sqrt{c+F_{\infty}}-\frac{1}{\sqrt{c+F_{\infty}}} w^{-1}-\frac{1}{2\left(c+F_{\infty}\right)^{3 / 2}} w^{-2}+\mathcal{O}\left(|w|^{-3}\right),
$$

\footnotetext{
${ }^{4}$ Dan Knopf has drawn to my attention a small oversight in a corresponding formula for $G$ in section 5 of 23 . As a consequence, a few gradient terms are missing in subsequent formulae of 23], yet these missing terms do not harm the argument made there.
} 
near $r=\infty$, remain the same; so, by Calabi's lemma, $\varphi$ gives rise to a Kähler metric on $\mathbb{C}^{n} \backslash\{0\}$ that can be extended smoothly to $M_{n}$.

We next settle the evolution equations of $\varphi$ and $\psi$ :

$$
\varphi_{t}=\frac{\varphi_{r r}}{\varphi_{r}}+(n-1) \frac{\varphi_{r}}{\varphi}-n,
$$

and

$$
\psi_{t}=\frac{\psi_{r r}}{\varphi_{r}}-\left(\frac{\varphi_{r r}}{\varphi_{r}^{2}}-\frac{n-1}{\varphi}\right) \psi_{r}-(n-1) \frac{\varphi_{r} \psi}{\varphi^{2}} .
$$

Since $\psi(\cdot, t) \equiv n-1$ on a neighborhood of $r=-\infty$, this last equation reduces to

$$
\psi_{t}=-(n-1)^{2} \frac{\varphi_{r}}{\varphi^{2}} .
$$

A further computation then shows that

$$
\left.\frac{\partial}{\partial t} \psi_{r}\right|_{t=0}=(n-1)^{2} \frac{e^{r}}{\varphi^{5}}\left(e^{r}-c\right),
$$

which, as before, is strictly negative for $r<\log c$. This concludes the proof of Theorem 3

Remark 7. More generally, for each $1 \leq k \leq n-1$, one can construct Kähler metrics with non-negative Ricci curvature on $k$-twisted bundles $\mathcal{F}_{k}^{n}$ that immediatly acquire mixed Ricci sign under Ricci flow. This is achieved by considering the potential $\varphi$ obtained when using the function $k a(r)$ instead of $a(r)$ and then using Calabi's lemma to glue back sections $S_{0}$ and $S_{\infty}$.

\section{ACKNOWLEDGMENTS}

I know of no adequate way of thanking my adviser, Dan Knopf, for his kind guidance and unwavering support. I am also thankful for the support received by the National Science Foundation under contract No. DMS-0545984. Finally, I extend my gratitude to Carlo Mantegazza and Wolfgang Ziller for support received during visits to the Centro di Ricerca Matematica Ennio De Giorgi and to the Instituto Nacional de Matemática Pura e Aplicada (IMPA), respectively, and to Huai-Dong Cao, Simon Brendle and Yanir A. Rubinstein for helpful comments on the original manuscript.

\section{REFERENCES}

1. Bando, Shigetoshi, On the classification of three-dimensional compact Kähler manifolds of non-negative bisectional curvature. J. Differential Geom. 19(2) (1984) 283-297. MR755227 (86i:53042)

2. Böhm, Christoph; Wilking, Burkhard, Nonnegatively curved manifolds with finite fundamental groups admit metrics with positive Ricci curvature. Geom. Funct. Anal. 17 (2007), no. 3, 665-681. MR2346271 (2008h:53050)

3. Böhm, Christoph; Wilking, Burkhard, Manifolds with positive curvature operators are space forms. Ann. of Math. (2) 167 (2008), no. 3, 1079-1097. MR2415394 (2009h:53146)

4. Brendle, Simon, A general convergence result for the Ricci flow in higher dimensions. Duke Math. J. 145 (2008), no. 3, 585-601. MR2462114(2010a:53132)

5. Brendle, Simon; Schoen, Richard, Manifolds with 1/4-pinched curvature are space forms. J. Amer. Math. Soc. 22 (2009), no. 1, 287-307. MR2449060(2010a:53045)

6. Calabi, Eugenio, Extremal Kähler metrics, Seminar on Differential Geometry, pp. 259-290, Ann. of Math. Stud., 102, Princeton Univ. Press, Princeton, NJ, 1982. MR645743(83i:53088) 
7. Cao, Huai-Dong, Existence of gradient Kähler-Ricci solitons. Elliptic and parabolic methods in geometry (Minneapolis, MN, 1994), 1-16, A K Peters, Wellesley, MA, 1996. MR 1417944 (98a:53058)

8. Cao, Huai-Dong, Limits of solutions to the Kähler-Ricci flow. J. Differential Geom. 45 (1997), no. 2, 257-272. MR.1449972 (99g:53042)

9. Cao, Huai-Dong, Deformation of Kähler metrics to Kähler-Einstein metrics on compact Kähler manifolds. Invent. Math. 81 (1985), no. 2, 359-372. MR799272 (87d:58051)

10. Cao, Huai-Dong; Hamilton, Richard S., On the preservation of positive orthogonal bisectional curvature under the Kähler-Ricci flow. Private communication (unpublished work) (1988).

11. Chen, Haiwen, Pointwise $\frac{1}{4}$-pinched 4-manifolds. Ann. Global Anal. Geom. 9 (1991), no. 2, 161-176. MR.1136125 (93b:53028)

12. Chen, Xiuxiong, On Kähler manifolds with positive orthogonal bisectional curvature. Adv. Math. 215 (2007), no. 2, 427-445. MR2355611 (2008h:32029)

13. Chen, Xiuxiong; Li, Haozhao, The Kähler-Ricci flow on Kähler manifolds with 2-non-negative traceless bisectional curvature operator. Chin. Ann. Math. Ser. B 29 (2008), no. 5, 543-556. MR2447486 (2010a:53133)

14. Chen, Xiuxiong; Tian, Gang, Ricci flow on Kähler-Einstein surfaces. Invent. Math. 147 (2002), no. 3, 487-544. MR.1893004 (2003c:53095)

15. Chen, Xiuxiong; Tian, Gang, Ricci flow on Kähler-Einstein manifolds. Duke Math. J. 131 (2006), no. 1, 17-73. MR2219236 (2007c:53083)

16. Chow, Bennett; Lu, Peng; Ni, Lei, Hamilton's Ricci flow. Graduate Studies in Mathematics, 77. American Mathematical Society, Providence, RI; Science Press, New York, 2006. MR2274812 (2008a:53068)

17. Chow, Bennett; Chu, Sun-Chin; Glickenstein, David; Guenther, Christine; Isenberg, James; Ivey, Tom; Knopf, Dan; Lu, Peng; Luo, Feng; Ni, Lei, The Ricci Flow: Techniques and Applications, Part I: Geometric Aspects. Mathematical Surveys and Monographs, 135. American Mathematical Society, Providence, RI, 2007. MR2302600 (2008f:53088)

18. Feldman, Mikhail; Ilmanen, Tom; Knopf, Dan, Rotationally symmetric shrinking and expanding gradient Kähler-Ricci solitons. J. Differential Geom. 65 (2003), no. 2, 169-209. MR.2058261 (2005e:53102)

19. Goldberg, Samuel I.; Kobayashi, Shoshichi, Holomorphic bisectional curvature. J. Differential Geometry 1 (1967), 225-233. MR0227901 (37:3485)

20. Hamilton, Richard S., Three-manifolds with positive Ricci curvature. J. Differential Geom. 17 (1982), no. 2, 255-306. MR664497 (84a:53050)

21. Hamilton, Richard S., Four-manifolds with positive curvature operator. J. Differential Geom. 24 (1986), no. 2, 153-179. MR.862046 (87m:53055)

22. Hamilton, Richard S., Four-manifolds with positive isotropic curvature. Comm. Anal. Geom. 5 (1997), no. 1, 1-92. MR 1456308 (99e:53049)

23. Knopf, Dan, Positivity of Ricci curvature under the Kähler-Ricci flow. Commun. Contemp. Math. 8 (2006), no. 1, 123-133. MR 2208813 (2006k:53114)

24. Koiso, Norihito, On rotationally symmetric Hamilton's equation for Kähler-Einstein metrics. Adv. Stud. Pure Math. (1990) 327-337. MR1145263 (93d:53057)

25. Nguyen, Huy T., Isotropic curvature and the Ricci flow. Int. Math. Res. Notices IMRN (2010), no. 3, 536-558. MR 2587576

26. Ni, Lei, Ricci flow and nonnegativity of sectional curvature. Math. Res. Lett. 11 (2004), no. 5-6, 883-904. MR2106247 (2005m:53123)

27. Mok, Ngaiming, The uniformization theorem for compact Kähler manifolds of non-negative holomorphic bisectional curvature. J. Differential Geom. 27(2) (1988) 179-214. MR925119 (89d:53115)

28. Phong, D. H.; Sturm, Jacob, On the Kähler-Ricci flow on complex surfaces. Pure Appl. Math. Q. 1 (2005), no. 2, part 1, 405-413. MR2194731 (2006h:53064)

29. Phong, D. H.; Sturm, Jacob, On stability and the convergence of the Kähler-Ricci flow. J. Differential Geom. 72 (2006), no. 1. MR2215459(2007a:53128)

30. Shi, Wan-Xiong, Complete noncompact three-manifolds with nonnegative Ricci curvature. J. Differential Geom. 29 (1989), 353-360. MR982179 (90c:53112)

31. Shi, Wan-Xiong, Deforming the metric on complete Riemannian manifolds. J. Differential Geom. 30 (1989), 223-301. MR1001277 (90i:58202) 
32. Shi, Wan-Xiong, Ricci flow and the uniformization on complete noncompact Kähler manifolds. J. Differential Geom. 45 (1997), 94-220. MR1443333 (98d:53099)

33. Wilking, Burkhard, Sharp estimates for the Ricci flow. International Symposium on Differential Geometry "In honor of Marcos Dajczer on his 60th birthday", 2009.

Department of Mathematics, University of Texas at Austin, Austin, Texas 78712

E-mail address: maximo@math.utexas.edu 